\title{
Sugammadex in the management of myasthenic patients undergoing surgery: beyond expectations
}

\author{
Michele Carron, Alessandro De Cassai, Federico Linassi \\ Department of Medicine-DIMED, Section of Anesthesiology and Intensive Care, University of Padova, Padova, Italy \\ Correspondence to: Michele Carron. Department of Medicine-DIMED, Section of Anesthesiology and Intensive Care, University of Padova, Via V. \\ Gallucci 13, 35121 Padova, Italy. Email: michele.carron@unipd.it. \\ Provenance: This is an invited article commissioned by the Academic Editor Dr. Hao Zhang, MD (Department of Anesthesiology, Rocket Force \\ Characteristic Medical Center of PLA, Beijing, China). \\ Comment on: Mouri H, Jo T, Matsui H, et al. Effect of sugammadex on postoperative myasthenic crisis in myasthenia gravis patients: propensity score \\ analysis of a Japanese nationwide database. Anesth Analg 2019. [Epub ahead of print].
}

Submitted Sep 21, 2019. Accepted for publication Oct 11, 2019.

doi: $10.21037 /$ atm.2019.10.35

View this article at: http://dx.doi.org/10.21037/atm.2019.10.35

Myasthenia gravis is an autoimmune disease characterized by antibodies that bind to acetylcholine receptors or functionally related molecules in the postsynaptic membrane of the neuromuscular junction. These antibodies induce skeletal muscle weakness that can be generalized or localized, is typically more severe in proximal muscles, and nearly always involves the eye, producing diplopia and ptosis. Muscle weakness improves with rest and worsens with activity (1). Myasthenia gravis is the most common primary disease of the neuromuscular junction, with an annual incidence of approximately 8 to 10 cases per 1 million persons and a prevalence of 150 to 250 cases per 1 million people (1). Acetylcholinesterase inhibitors, with or without concurrent immunosuppressive therapy, represent first-line treatment for the disease. Thymectomy should be considered in patients with a thymoma and myasthenia gravis (1).

In a patient with myasthenia gravis, two kinds of crises may develop, both causing weakness, sometimes difficult to differentiate: cholinergic crisis or myasthenic crisis. Cholinergic crises are generally caused by an excess of cholinesterase inhibitor medications. They produce symptoms of cholinergic overactivity, such as hypersalivation, sweating, abdominal cramps, urinary urgency, bradycardia, muscle fasciculations, and muscle weakness. Myasthenic crises can be considered disease exacerbations, which may be triggered by several factors, including infection, emotional stress, pregnancy, and certain medications (e.g., verapamil, fluoroquinolones, macrolides, aminoglycosides) (1,2). Myasthenic crises are responsible for delayed extubation after surgery and a high incidence of postoperative complications in patients with myasthenia gravis. Kas and colleagues reported successful extubation in the operating room in only $5.2 \%$ of 324 myasthenic patients undergoing transsternal thymectomy; $29.6 \%$, $45.6 \%$, and $37.3 \%$ of the patients required ventilatory support for 24, 48, and 72 hours or more, respectively (3). Major complications (e.g., respiratory failure, pneumonia, heart failure) occurred in $23.7 \%$ of the patients, and minor complications (e.g., cardiac dysrhythmia, retention of airway secretions, tracheobronchitis) were noted in $65 \%$. Specifically, respiratory failure developed in $16.3 \%$ of patients after simple thymectomy, $19.3 \%$ of patients after thymoma removal, and in $30.3 \%$ of patients after extended thymectomy (3). Similarly, Leuzzi and colleagues reported successful extubation in the operating room in only $4.5 \%$ of myasthenic patients after thymectomy (4).

Anesthetic drugs may contribute to the development of a perioperative myasthenic crisis (2). Neuromuscularblocking agents (NMBAs) are especially problematic, as patients with myasthenia gravis are particularly sensitive to these drugs $(1,2)$. The anesthetic approach is often modified to avoid or limit the use of NMBAs in these patients. Gritti and colleagues reported that increasing the percentage of patients receiving general (propofol, sevoflurane or desflurane) anesthesia without NMBA from $67 \%$ to $94 \%$ increased the rate of patients transferred to the surgical 
ward after surgery from $26.0 \%$ to $93.2 \%$, significantly reducing intensive care unit (ICU) admission rates (5). Similarly, Fujita and colleagues reported that thymectomy was successfully performed in $90.9 \%$ of patients receiving combined general (sevoflurane) and epidural anesthesia without NMBAs, and the percentage of patients not extubated in the operating room because of respiratory depression or other reasons was lower in patients who did not receive NMBAs $(28.3 \%)$ than in those who received NMBAs (50\%) (6). In a study of 122 thymectomies performed under combined general (sevoflurane) and epidural anesthesia without NMBAs, Watanabe and colleagues reported that $11.5 \%$ of patients developed a postoperative myasthenic crisis, requiring reintubation after failed extubation and/or prolonged ventilator support for more than 48 hours postoperatively (7). Thus, anesthesia per se can trigger factor a myasthenic crisis, but the risk of a crisis is clearly increased with the use of NMBAs (1-7). Although avoidance of NMBAs is recommended, this is not always possible (5-7); NMBAs are particularly advised for laparoscopic surgery (2).

Sugammadex has changed the management of intraoperative neuromuscular blockade $(\mathrm{NMB})$ in patients with myasthenia gravis (2). Sugammadex is a modified $\gamma$-cyclodextrin that reverses the effects of steroidal NMBAs. It is most commonly used for rocuronium reversal at the end of surgery. After intravenous injection, sugammadex initially acts by encapsulating and inactivating unbound rocuronium circulating in the plasma to form tight 1:1 complexes that are excreted in the urine. Secondly, sugammadex promotes the dissociation of rocuronium from neuromuscular junctions by creating a concentration gradient from the neuromuscular junction to the plasma, where it is subsequently encapsulated, inactivated, and excreted. Sugammadex does not affect the release or breakdown of acetylcholine, and it does not interfere with the morphology or physiology of the neuromuscular junction. So, when used for reversing NMB, sugammadex is not accompanied by the risk of triggering a cholinergic crisis, which may occur with cholinesterase inhibitors. Several case reports and series have described the potential benefits of a rocuronium-sugammadex strategy for neuromuscular block management in myasthenic patients undergoing intravenous or inhalational general anesthesia (Table 1) $(8-25)$. In the majority of reports, use of sugammadex was associated with fast, complete reversal of rocuroniuminduced NMB, as well as successful extubation at the end of surgery and no postoperative complications (8-25).

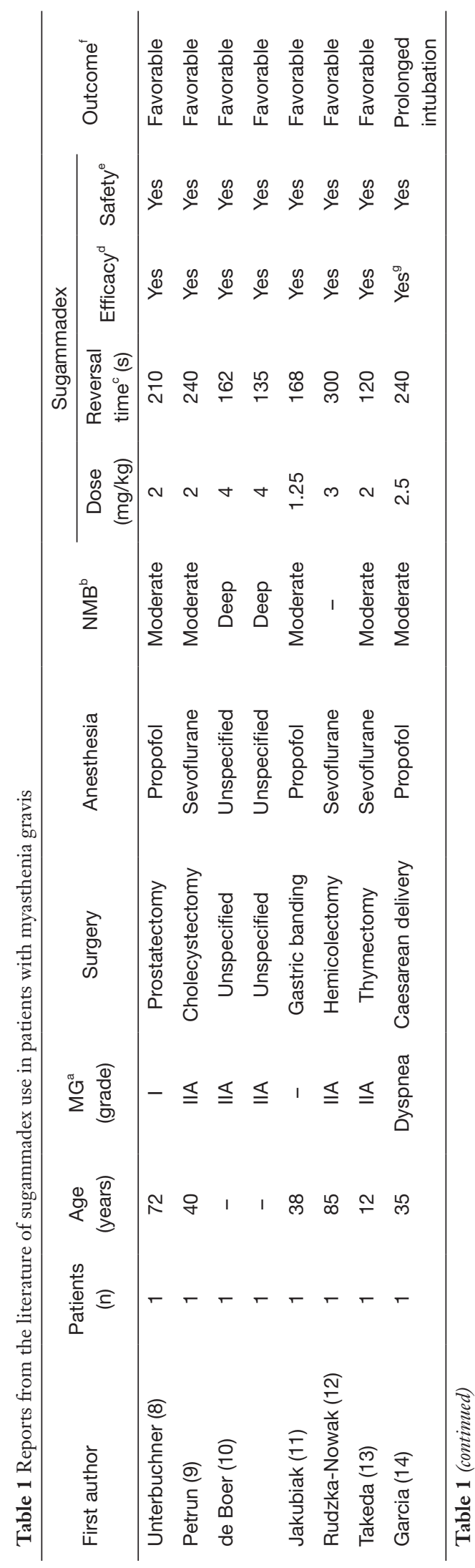







The few reported cases of incomplete recovery [trainof-four (TOF) ratio $<0.9$ during neuromuscular function monitoring] after sugammadex and concomitant muscle weakness $(14,15,17,25)$ were successfully managed after administration of acetylcholinesterase inhibitors $(15,17,25)$. However, there is a paucity of studies comparing the effects of sugammadex versus acetylcholinesterase inhibitors on perioperative outcomes of patients with myasthenia gravis.

In an article published on May 20, 2019 in Anesthesia \& Analgesia, Mouri and colleagues reported the results of their retrospective cohort analysis of 795 adults with myasthenia gravis who underwent thymectomy under general anesthesia from July 1, 2010 to March 31, 2016 (26). The patients were selected from the Japanese Diagnosis Procedure Combination nationwide database. Patients who received sugammadex were compared to a control group of patients who did not receive sugammadex; the authors did not specify the reversal agent (if any) used in the control group. The primary outcome was postoperative myasthenic crisis, which was defined as respiratory failure necessitating prolonged ( $\geq 3$ days) mechanical ventilation postoperatively or reintubation in the first 30 days postthymectomy. The secondary outcomes were the occurrence of postoperative pneumonia or tracheostomy, 28-day mortality, hospital length of stay after surgery, and total hospitalization costs (26). The main result was that, compared to control group patients who did not receive sugammadex $(\mathrm{n}=288)$, patients managed with rocuronium-sugammadex $(n=507)$ had a significantly lower risk of postoperative myasthenic crisis $[4.3 \%$ vs. $8.7 \%$; odds ratio (OR), 0.48 ; $95 \%$ confidence interval (CI), 0.25-0.91] (26). Unfortunately, the authors did not indicate whether the postoperative myasthenic crises were the result of failure to adequately reverse rocuroniuminduced NMB by sugammadex (26). Based on the literature, approximately $98 \%$ of patients with myasthenia gravis treated with sugammadex underwent successful tracheal extubation at the end of surgery after reaching full recovery from $\mathrm{NMB}$ (documented by a TOF ratio $>0.9$ ), avoiding postoperative ICU admission for mechanical ventilation (8-25).

It is important to note that although sugammadex may avoid muscle weakness related to the residual effects of NMBAs, it may not prevent exacerbation of the underlying myasthenia gravis after surgery. Severity of the disease itself is associated with an increased risk of postoperative myasthenic crisis. In multivariate logistic regression analysis, Leuzzi and colleagues showed that Osserman stage IIB (OR, $5.69)$ and III-IV (OR, 11.33), body mass index $>28 \mathrm{~kg} / \mathrm{m}^{2}$ (OR, 3.65), previous myasthenic crisis (OR, 24.10), duration of symptoms $>2$ years $(\mathrm{OR}, 5.94)$, and lung resection $(\mathrm{OR}$,
8.48) were all independent risk factors for the development of a postoperative myasthenic crisis (4). When a myasthenic crisis occurs, administration of an acetylcholinesterase inhibitor, such as pyridostigmine or neostigmine $(1,2)$, seems to improve muscle weakness after general anesthesia $(14,15,17,25)$. Intravenous immune globulin or plasma exchange are other options suggested for persistent severe myasthenic crises (1).

The study of Mouri and colleagues was unable to demonstrate a significant decrease in postoperative pneumonia with sugammadex, compared to the control group (1.0\% vs. $2.4 \%$, respectively; OR, 0.44; $95 \%$ CI, $0.17-1.14)$ (26). Previous reports in non-myasthenic patients have shown that use of NMBAs increases the risk of pneumonia, and reversal of NMB reduces this risk. Bulka and colleagues reported that surgical patients receiving an NMBA had a higher absolute incidence of postoperative pneumonia (9.00 vs. 5.22 per 10,000 persondays at risk), with a significantly increased incidence rate ratio of 1.79 (27). Patients who received an NMBA but no reversal agent were 2.26 times more likely to develop postoperative pneumonia than patients who received an NMBA and neostigmine (27). Appropriate monitoring of neuromuscular function and reversal are thereby recommended to minimize the risk of complications related to residual $\mathrm{NMB}$, including postoperative pneumonia (28). In a meta-analysis of randomized controlled trials involving patients without myasthenia gravis, our group noted that sugammadex was associated with a significantly lower risk of postoperative respiratory adverse events $(\mathrm{OR}, 0.36)$ and weakness (OR, 0.45), compared to neostigmine (28). The Mouri and colleagues' study is the first study providing evidence in support of the potential benefits of sugammadex over neostigmine in reducing the risk of postoperative pneumonia, although the favorable trend did not reach statistical significance (26).

Interestingly, the study of Mouri and colleagues showed that use of sugammadex reduced median length of hospital stay after surgery (10 vs. 11 days; $\mathrm{P}<0.001)$ and total hospitalization costs $(\$ 13,186$ vs. $\$ 14,119 ; \mathrm{P}<0.001)$, compared with non-use of sugammadex (26). Although sugammadex produces faster and more predictable recovery from NMB than neostigmine, the direct cost of sugammadex is higher. Cost-effectiveness analyses have demonstrated that using sugammadex to reduce the time to full reversal of NMB in the operating room can be economically beneficial, depending on the cost of the operating room, the actual time saved by using 
sugammadex, and whether this saved time is used productively (29-31). In addition to enhancing operating room efficiency by accelerating transfer from the operating room, use of sugammadex may also reduce overall costs by decreasing the risk of postoperative complications and unplanned ICU admissions (30). Furthermore, Ledowski and colleagues noted that sugammadex use reduced the length of hospital stay by several hours ( 73 vs. $78 \mathrm{~h} ; \mathrm{P}=0.044$ ) in nonmyasthenic patients and suggested that this may contribute to economic benefits if it avoids an additional night in the hospital (with an estimated average cost of US \$420) (32). Thus, it is not surprising that Mouri and colleagues found a significant reduction in total hospitalization costs with sugammadex. Oh and colleagues previously reported that sugammadex reduced total hospital charges by $24 \%$ in nonmyasthenic patients undergoing major abdominal surgery, compared with neostigmine (33). In that study, sugammadex was associated with a $20 \%$ reduction in hospital length of stay and a 34\% reduction in 30-day unplanned readmission rate. Readmission data were not reported in the Mouri and colleagues' study (26). Whether sugammadex results in further potential economic benefit in patients with myasthenia gravis will depend on readmission costs and the extent of reduction in 30-day unplanned readmission rates in these patients (34).

The study by Mouri and colleagues leaves us with some important messages. Sugammadex is superior to neostigmine for reversing rocuronium-induced $\mathrm{NMB}$ in patients with myasthenia gravis undergoing surgery. It represents the treatment of choice for reducing the risk of perioperative myasthenic crisis, and possibly decreasing the risk of postoperative pneumonia, in these patients. Given the current high costs of medical care, the overall economic benefits of sugammadex represent a welcome addition to the armamentarium of anesthesiologists.

\section{Acknowledgments}

None.

\section{Footnote}

Conflicts of Interest: M Carron has received payments for lectures from Merck Sharp \& Dohme (MSD), Rome, Italy. A De Cassai and F Linassi have no conflicts of interest to declare.

Ethical Statement: The authors are accountable for all aspects of the work in ensuring that questions related to the accuracy or integrity of any part of the work are appropriately investigated and resolved.

\section{References}

1. Gilhus NE. Myasthenia gravis. N Engl J Med 2016;375:2570-81.

2. Sungur $Z$, Sentürk $M$. Anaesthesia for thymectomy in adult and juvenile myasthenic patients. Curr Opin Anaesthesiol 2016;29:14-9.

3. Kas J, Kiss D, Simon V, et al. Decade-long experience with surgical therapy of myasthenia gravis: early complications of 324 transsternal thymectomies. Ann Thorac Surg 2001;72:1691-7.

4. Leuzzi G, Meacci E, Cusumano G, et al. Thymectomy in myasthenia gravis: proposal for a predictive score of postoperative myasthenic crisis. Eur J Cardiothorac Surg 2014;45:e76-88; discussion e88.

5. Gritti P, Sgarzi M, Carrara B, et al. A standardized protocol for the perioperative management of myasthenia gravis patients. Experience with 110 patients. Acta Anaesthesiol Scand 2012;56:66-75.

6. Fujita Y, Moriyama S, Aoki S, et al. Estimation of the success rate of anesthetic management for thymectomy in patients with myasthenia gravis treated without muscle relaxants: a retrospective observational cohort study. J Anesth 2015;29:794-7.

7. Watanabe A, Watanabe T, Obama T, et al. Prognostic factors for myasthenic crisis after transsternal thymectomy in patients with myasthenia gravis. J Thorac Cardiovasc Surg 2004;127:868-76.

8. Unterbuchner C, Fink H, Blobner M. The use of sugammadex in a patient with myasthenia gravis. Anaesthesia 2010;65:302-5.

9. Petrun AM, Mekis D, Kamenik M. Successful use of rocuronium and sugammadex in a patient with myasthenia. Eur J Anaesthesiol 2010;27:917-8.

10. de Boer HD, van Egmond J, Driessen JJ, et al. Sugammadex in patients with myasthenia gravis. Anaesthesia 2010;65:653.

11. Jakubiak J, Gaszyński T, Gaszyński W. Neuromuscular block reversal with sugammadex in a morbidly obese patient with myasthenia gravis. Anaesthesiol Intensive Ther 2012;44:28-30.

12. Rudzka-Nowak A, Piechota M. Anaesthetic management of a patient with myasthenia gravis for abdominal surgery using sugammadex. Arch Med Sci 2011;7:361-4. 
13. Takeda A, Kawamura M, Hamaya I, et al. Case of anesthesia for thoracoscopic thymectomy in a pediatric patient with myasthenia gravis: reversal of rocuronium-induced neuromuscular blockade with sugammadex. Masui 2012;61:855-8.

14. Garcia V, Diemunsch P, Boet S. Use of rocuronium and sugammadex for caesarean delivery in a patient with myasthenia gravis. Int J Obstet Anesth 2012;21:286-7.

15. Kiss G, Lacour A, d'Hollander A. Fade of train-of-four ratio despite administration of more than $12 \mathrm{mg} \mathrm{kg}(-1)$ sugammadex in a myasthenia gravis patient receiving rocuronium. Br J Anaesth 2013;110:854-5.

16. Sungur Ulke Z, Yavru A, Camci E, et al. Rocuronium and sugammadex in patients with myasthenia gravis undergoing thymectomy. Acta Anaesthesiol Scand 2013;57:745-8.

17. Sugi Y, Nitahara K, Shiroshita T, et al. Restoration of train-of-four ratio with neostigmine after insufficient recovery with sugammadex in a patient with myasthenia gravis. A A Case Rep 2013;1:43-5.

18. Casarotti P, Mendola C, Cammarota G, et al. High-dose rocuronium for rapid-sequence induction and reversal with sugammadex in two myasthenic patients. Acta Anaesthesiol Scand 2014;58:1154-8.

19. de Boer HD, Shields MO, Booij LH. Reversal of neuromuscular blockade with sugammadex in patients with myasthenia gravis: a case series of 21 patients and review of the literature. Eur J Anaesthesiol 2014;31:715-21.

20. Vymazal T, Krecmerova M, Bicek V, et al. Feasibility of full and rapid neuromuscular blockade recovery with sugammadex in myasthenia gravis patients undergoing surgery - a series of 117 cases. Ther Clin Risk Manag 2015;11:1593-6.

21. Kim RK, Kim SY. Rapid return of spontaneous respiration after general anesthesia with sugammadex in a patient with myasthenia gravis. J Lifestyle Med 2016;6:43-6.

22. Dabbous AS, Nehme PW, Abou Leila AM. Anesthetic management of aortic valve replacement in a myasthenia gravis patient, the era of a new reversal. Middle East J Anaesthesiol 2016;23:491-4.

23. Shah D, Dharmarajah A. Use of sugammadex in an octagenerian with myaesthenia gravis undergoing emergency laporotomy. J Clin Anesth 2017;37:109-10.

24. Kondo M, Yoshikawa Y, Terada H, et al. Anesthetic management of total aortic arch replacement in a myasthenia gravis patient under deep hypothermic circulatory arrest. Case Rep Anesthesiol 2019;2019:3278147.

25. Fernandes HDS, Ximenes JLS, Nunes DI, et al. Failure of reversion of neuromuscular block with sugammadex in patient with myasthenia gravis: case report and brief review of literature. BMC Anesthesiol 2019;19:160.

26. Mouri H, Jo T, Matsui H, et al. Effect of sugammadex on postoperative myasthenic crisis in myasthenia gravis patients: propensity score analysis of a Japanese Nationwide Database. Anesth Analg 2019. [Epub ahead of print].

27. Bulka CM, Terekhov MA, Martin BJ, et al. Nondepolarizing neuromuscular blocking agents, reversal, and risk of postoperative pneumonia. Anesthesiology 2016;125:647-55.

28. Carron M, Zarantonello F, Tellaroli P, et al. Efficacy and safety of sugammadex compared to neostigmine for reversal of neuromuscular blockade: a meta-analysis of randomized controlled trials. J Clin Anesth 2016;35:1-12.

29. Paton F, Paulden M, Chambers D, et al. Sugammadex compared with neostigmine/glycopyrrolate for routine reversal of neuromuscular block: a systematic review and economic evaluation. Br J Anaesth 2010;105:558-67.

30. Carron M, Baratto F, Zarantonello F, et al. Sugammadex for reversal of neuromuscular blockade: a retrospective analysis of clinical outcomes and cost-effectiveness in a single center. Clinicoecon Outcomes Res 2016;8:43-52.

31. Insinga RP, Joyal C, Goyette A, et al. A discrete event simulation model of clinical and operating room efficiency outcomes of sugammadex versus neostigmine for neuromuscular block reversal in Canada. BMC Anesthesiol 2016;16:114.

32. Ledowski T, Hillyard S, Kozman A, et al. Unrestricted access to sugammadex: impact on neuromuscular blocking agent choice, reversal practice and associated healthcare costs. Anaesth Intensive Care 2012;40:340-3.

33. Oh TK, Oh AY, Ryu JH, et al. Retrospective analysis of 30-day unplanned readmission after major abdominal surgery with reversal by sugammadex or neostigmine. Br J Anaesth 2019;122:370-8.

34. Carron M, De Cassai A, Ieppariello G. Reversal of rocuronium-induced neuromuscular block: is it time for sugammadex to replace neostigmine? Br J Anaesth 2019;123:e157-9.

Cite this article as: Carron M, De Cassai A, Linassi F. Sugammadex in the management of myasthenic patients undergoing surgery: beyond expectations. Ann Transl Med 2019;7(Suppl 8):S307. doi: 10.21037/atm.2019.10.35 